T. Halverson, M. Mazzocco and A. Ram

Nagoya Math. J.

Vol. 195 (2009), 125-152

\title{
COMMUTING FAMILIES IN HECKE AND TEMPERLEY-LIEB ALGEBRAS
}

\author{
TOM HALVERSON, MANUELA MAZZOCCO AND ARUN RAM
}

\begin{abstract}
We define analogs of the Jucys-Murphy elements for the affine Temperley-Lieb algebra and give their explicit expansion in terms of the basis of planar Brauer diagrams. These Jucys-Murphy elements are a family of commuting elements in the affine Temperley-Lieb algebra, and we compute their eigenvalues on the generic irreducible representations. We show that they come from Jucys-Murphy elements in the affine Hecke algebra of type A, which in turn come from the Casimir element of the quantum group $U_{h} \mathfrak{g l}_{n}$. We also give the explicit specializations of these results to the finite Temperley-Lieb algebra.
\end{abstract}

\section{$\S 1$. Introduction}

The "Jucys-Murphy elements" are a family of commuting elements in the group algebra of the symmetric group. In characteristic 0 , these elements have enough distinct eigenvalues to give a full analysis of the representation theory of the symmetric group [OV]. Even in positive characteristic these elements are powerful tools $[\mathrm{K}]$. Similar elements are used in the Hecke algebras of type A and, in a strong sense, it is these elements that control the beautiful connections between the modular representation theory of Hecke algebras of type A and the Fock space representations of the affine quantum group (see $[\mathrm{Ar}]$ and $[\mathrm{Gr}]$ ).

Since the Temperley-Lieb algebra is a quotient of the Hecke algebra of type A it inherits a commuting family of elements from the Hecke algebra (these elements can be viewed as the image, under a surjective homomorphism, of the commutative subalgebra in the Bernstein presentation of the affine Hecke algebra). In order to use these elements for modular representation theory of the Temperley-Lieb algebra it is important to have good

Received October 5, 2007.

Revised November 18, 2008.

Accepted February 4, 2009.

2000 Mathematics Subject Classification: Primary 20G05; Secondary 16G99, 81R50, $82 \mathrm{~B} 20$. 
control of their expansion in terms of the standard basis of noncrossing diagrams. In this paper we study this question, in the more general setting of the affine Temperley-Lieb algebras. Specifically, we analyze a convenient choice of a commuting family of elements in the affine Temperley-Lieb algebra. Our main result, Theorem 2.9, is an explicit expansion of these elements in the standard basis. The fact that, in the Templerley-Lieb algebra, these elements have integral coefficients is made explicit in Remark 2.10. The import of this result is that this commuting family can be used to attack questions in modular representation theory.

In Section 3 we review the Schur-Weyl duality setup of Orellana and Ram [OR] which (following the ideas in [Re]) explains how commuting families in centralizer algebras arise naturally from Casimir elements. We explain, in detail, the cases that lead to commuting families in the affine Hecke algebras of type A and the affine Temperley-Lieb algebra. One new consequence of our analysis is an explanation of the "special" relation that is used in one of the Temperley-Lieb algebras of Graham and Lehrer [GL2]. In our context, this relation appears naturally from the Schur-Weyl duality (see Proposition 3.2). Using the knowledge of eigenvalues of Casimir elements we compute the eigenvalues of the commuting families in the affine Hecke algebra and in the affine Temperley-Lieb algebra in the generic irreducible representations (analogues of the Specht, or Weyl, modules).

The representation theory of Temperley-Lieb algebras and affine Temperley-Lieb algebras is 'well understood'. In particular, in the paper [GL1], the cell modules and the irreducible modules of the affine TemperleyLieb algebra are analyzed in detail and the eigenvalues of a commuting family of 'Murphy elements' on these modules are determined. They show ([GL1, Thm. (9.6)]) that the representations of the affine Hecke algebra which appear as affine Temperley-Lieb algebra representations are those representations that correspond, under the Deligne-Langlands correspondence [KL], to unipotent elements in $G L_{n}$ with at most two Jordan blocks. The commuting family of elements we use in this paper does not differ significantly from those used in [GL1]. Our results provide an expansion of these elements in terms of the basis of noncrossing diagrams and place the representations studied in [GL1] into a Schur-Weyl duality context.

The research of M. Mazzocco was partially supported by a Mark Mensink Honors Research Award at the University of Wisconsin, Madison. She was an undergraduate researcher participating in research and teaching initiatives partly supported by the National Science Foundation under 
grant DMS-0353038. The research of A. Ram was also partially supported by this award. A significant portion of this research was done during a residency of A. Ram at the Max-Planck-Institut für Mathematik (MPI) in Bonn. He thanks the MPI for support, hospitality, and a wonderful working environment. The research of T. Halverson was partially supported by the National Science Foundation under grant DMS-0100975.

\section{§2. Affine braid groups, Hecke and Temperley-Lieb algebras}

\subsection{The affine braid group $\tilde{\mathcal{B}}_{k}$}

The affine braid group is the group $\tilde{\mathcal{B}}_{k}$ of affine braids with $k$ strands (braids with a flagpole). The group $\tilde{\mathcal{B}}_{k}$ is presented by generators $T_{1}, T_{2}, \ldots$, $T_{k-1}$ and $X^{\varepsilon_{1}}$,
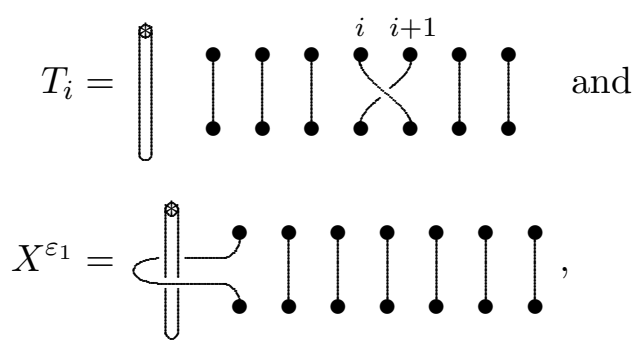

with relations

$$
\begin{array}{ll}
X^{\varepsilon_{1}} T_{1} X^{\varepsilon_{1}} T_{1}=T_{1} X^{\varepsilon_{1}} T_{1} X^{\varepsilon_{1}} & \\
X^{\varepsilon_{1}} T_{i}=T_{i} X^{\varepsilon_{1}}, & \text { for } i>1, \\
T_{i} T_{j}=T_{j} T_{i}, & \text { if }|i-j|>1, \\
T_{i} T_{i+1} T_{i}=T_{i+1} T_{i} T_{i+1}, & \text { if } 1 \leq i \leq k-2 .
\end{array}
$$

For $1 \leq i \leq k$ define

$$
\begin{aligned}
& X^{\varepsilon_{i}}=T_{i-1} T_{i-2} \cdots T_{2} T_{1} X^{\varepsilon_{1}} T_{1} T_{2} \cdots T_{i-1}
\end{aligned}
$$

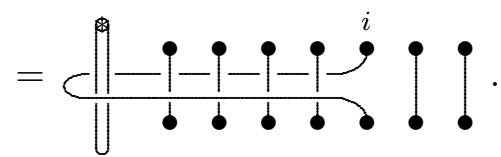

By drawing pictures of the corresponding affine braids it is easy to check that

$$
X^{\varepsilon_{i}} X^{\varepsilon_{j}}=X^{\varepsilon_{j}} X^{\varepsilon_{i}}, \quad \text { for } 1 \leq i, j \leq k,
$$


so that the elements $X^{\varepsilon_{1}}, \ldots, X^{\varepsilon_{k}}$ are a commuting family for $\tilde{\mathcal{B}}_{k}$. Thus $X=\left\langle X^{\varepsilon_{i}} \mid 1 \leq i \leq k\right\rangle$ is an abelian subgroup of $\tilde{\mathcal{B}}_{k}$. The free abelian group generated by $\varepsilon_{1}, \ldots, \varepsilon_{k}$ is $\mathbb{Z}^{k}$ and

$$
X=\left\{X^{\lambda} \mid \lambda \in \mathbb{Z}^{k}\right\} \quad \text { where } \quad X^{\lambda}=\left(X^{\varepsilon_{1}}\right)^{\lambda_{1}}\left(X^{\varepsilon_{2}}\right)^{\lambda_{2}} \cdots\left(X^{\varepsilon_{k}}\right)^{\lambda_{k}},
$$

for $\lambda=\lambda_{1} \varepsilon_{1}+\cdots+\lambda_{k} \varepsilon_{k}$ in $\mathbb{Z}^{k}$.

Remark 2.1. An alternate presentation of $\tilde{\mathcal{B}}_{k}$ can be given using the generators $T_{0}, T_{1}, \ldots, T_{k-1}$ and $\tau$ where

$$
\tau=X^{-\varepsilon_{1}} T_{1}^{-1} \cdots T_{k-1}^{-1}=
$$

Remark 2.2. The affine braid group $\tilde{\mathcal{B}}_{k}$ is the affine braid group of type $G L_{k}$. The affine braid groups of type $S L_{k}$ and $P G L_{k}$ are the subgroup

$$
\tilde{\mathcal{B}}_{Q}=\left\langle T_{0}, T_{1}, \ldots, T_{k-1}\right\rangle \quad \text { and the quotient } \quad \tilde{\mathcal{B}}_{P}=\frac{\tilde{\mathcal{B}}_{k}}{\left\langle\tau^{k}\right\rangle}, \quad \text { respectively. }
$$

Then $\tau^{k}=X^{-\varepsilon_{1}} X^{-\varepsilon_{2}} \cdots X^{-\varepsilon_{k}}$ is a central element of $\tilde{\mathcal{B}}_{k}, \tau T_{i} \tau^{-1}=T_{i+1}$ (where the indices are taken $\bmod k$ ), and

$$
Z\left(\tilde{\mathcal{B}}_{k}\right)=\left\langle\tau^{k}\right\rangle, \quad \tilde{\mathcal{B}}_{k}=\langle\tau\rangle \ltimes \tilde{\mathcal{B}}_{Q}, \quad \tilde{\mathcal{B}}_{P}=\langle\bar{\tau}\rangle \ltimes \tilde{\mathcal{B}}_{Q}
$$

In $\tilde{\mathcal{B}}_{k}$ we have $\langle\tau\rangle \cong \mathbb{Z}$, and $\bar{\tau} \in \tilde{\mathcal{B}}_{P}$ is defined to be the image of $\tau$ under the homomorphism $\mathbb{Z} \rightarrow \mathbb{Z} / k \mathbb{Z}$ so that $\langle\bar{\tau}\rangle \cong \mathbb{Z} / k \mathbb{Z}$.

\subsection{The Temperley-Lieb algebra $T L_{k}(n)$}

A Temperley-Lieb diagram on $k$ dots is a graph with $k$ dots in the top row, $k$ dots in the bottom row, and $k$ edges pairing the dots such that the graph is planar (without edge crossings). For example,

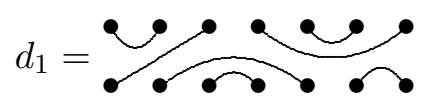

and

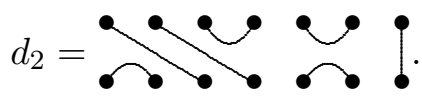


are Temperley-Lieb diagrams on 7 dots. The composition $d_{1} \circ d_{2}$ of two diagrams $d_{1}, d_{2} \in T_{k}$ is the diagram obtained by placing $d_{1}$ above $d_{2}$ and identifying the bottom vertices of $d_{1}$ with the top dots of $d_{2}$ removing any connected components that live entirely in the middle row. If $T_{k}$ is the set of Temperley-Lieb diagrams on $k$ dots then the Temperley-Lieb algebra $T L_{k}(n)$ is the associative algebra with basis $T_{k}$,

$T L_{k}(n)=\operatorname{span}\left\{d \in T_{k}\right\}$ with multiplication defined by $d_{1} d_{2}=n^{\ell}\left(d_{1} \circ d_{2}\right)$,

where $\ell$ is the number of blocks removed from the middle row when constructing the composition $d_{1} \circ d_{2}$ and $n$ is a fixed element of the base ring. For example, using the diagrams $d_{1}$ and $d_{2}$ above, we have

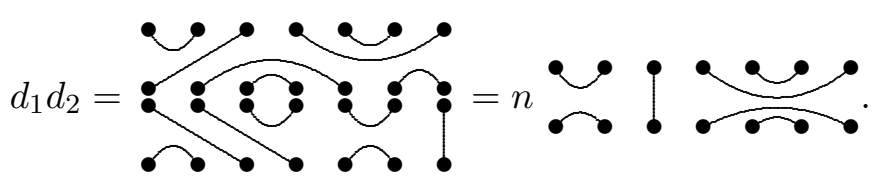

The algebra $T L_{k}(n)$ is presented by generators

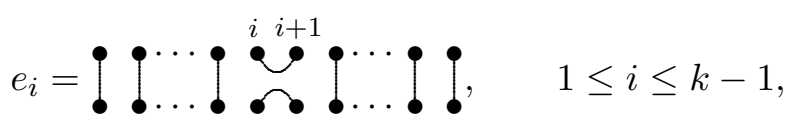

and relations

$$
e_{i}^{2}=n e_{i}, \quad e_{i} e_{i \pm 1} e_{i}=e_{i}, \quad \text { and } \quad e_{i} e_{j}=e_{j} e_{i}, \quad \text { if }|i-j|>1
$$

(see [GHJ, Lemma 2.8.4]).

Remark 2.3. In the definition of the Temperley-Lieb algebra, and for other algebras defined in this paper, the base ring could be any one of several useful rings (e.g. $\mathbb{C}, \mathbb{C}(q), \mathbb{C}[[h]], \mathbb{Z}\left[q, q^{-1}\right], \mathbb{Z}[n]$ or localizations of these at special primes). The most useful approach is to view the results of computations as valid over any ring $R$ with $n, q, h \in R$ such that the formulas make sense.

\subsection{The surjection $\tilde{H}_{k}(q) \mapsto T L_{k}(n)$}

The affine Hecke algebra $\tilde{H}_{k}$ is the quotient of the group algebra of the affine braid group $\mathbb{C} \tilde{\mathcal{B}}_{k}$ by the relations

$$
T_{i}^{2}=\left(q-q^{-1}\right) T_{i}+1, \quad \text { so that } \mathbb{C} \tilde{\mathcal{B}}_{k} \longrightarrow \tilde{H}_{k}
$$


is a surjective homomorphism ( $q$ is a fixed element of the base ring). The affine Hecke algebra $\tilde{H}_{k}$ is the affine Hecke algebra of type $G L_{k}$. The affine Hecke algebras of types $S L_{k}$ and $P G L_{k}$ are, respectively, the quotients $\tilde{H}_{Q}$ and $\tilde{H}_{P}$ of the group algebras of $\tilde{\mathcal{B}}_{Q}$ and $\tilde{\mathcal{B}}_{P}$ (see Remark 2.2) by the relations (2.8).

The Iwahori-Hecke algebra is the subalgebra $H_{k}$ of $\tilde{H}_{k}$ generated by $T_{1}, \ldots, T_{k-1}$. In the Iwahori-Hecke algebra $H_{k}$, define

$$
e_{i}=q-T_{i}, \quad \text { for } i=1,2, \ldots, k-1 .
$$

Direct calculations show that $e_{i}^{2}=\left(q+q^{-1}\right) e_{i}$ and that $e_{1} e_{2} e_{1}=e_{1}$ and $e_{2} e_{1} e_{2}=e_{2}$ if and only if

$$
q^{3}-q^{2} T_{1}-q^{2} T_{2}+q T_{1} T_{2}+q T_{2} T_{1}-T_{1} T_{2} T_{1}=0 .
$$

Thus, setting $n=[2]=q+q^{-1}$, there are surjective algebra homomorphisms given by

$$
\begin{aligned}
& \psi: \tilde{H}_{k}(q) \longrightarrow H_{k}(q) \longrightarrow T L_{k}(n) \\
& X^{\varepsilon_{1}} \longmapsto 1 \longmapsto 1 \\
& T_{i} \longmapsto T_{i} \longmapsto q-e_{i} .
\end{aligned}
$$

The kernel of $\psi$ is generated by the element on the left hand side of equation (2.10). In the notation of Theorem 4.1, the representations of $H_{k}$ correspond to the case when $\mu=\emptyset$. Writing $\tilde{H}_{k}^{\lambda / \emptyset}$ as $\tilde{H}_{k}^{\lambda}$, the element from (2.10) acts as 0 on the irreducible Iwahori-Hecke algebra modules $\tilde{H}_{3}^{\mathbf{m}}$ and $\tilde{H}_{3}^{\mathbf{P}}$, and (up to a scalar multiple) it is a projection onto $\tilde{H}_{3}^{\mathrm{⿴}}$.

Remark 2.4. There is an alternative surjective homomorphism that instead sends $T_{i} \mapsto e_{i}-q^{-1}$. This alternative surjection has kernel generated by

$$
q^{-3}+q^{-2} T_{1}+q^{-2} T_{2}+q^{-1} T_{1} T_{2}+q^{-1} T_{2} T_{1}+T_{2} T_{1} T_{2} .
$$

This element is 0 on $\tilde{H}_{3}^{\mathrm{Q}}$ and $\tilde{H}_{3}^{\mathrm{P}}$, and (up to a scalar multiple) it is a

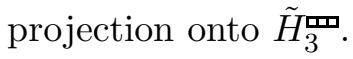

Remark 2.5. A priori, there are two different kinds of integrality for the Temperley-Lieb algebra: coefficients in $\mathbb{Z}[n]$ or coefficients in $\mathbb{Z}\left[q, q^{-1}\right]$ (in terms of the basis of Temperley-Lieb diagrams). The relation between these is as follows. If

$$
[2]=q+q^{-1}=n \quad \text { then } \quad q=\frac{1}{2}\left(n+\sqrt{n^{2}-4}\right), \quad q^{-1}=\frac{1}{2}\left(n-\sqrt{n^{2}-4}\right),
$$


since $q^{2}-n q+1=0$. Then

$$
[k]=\frac{q^{k}-q^{-k}}{q-q^{-1}}=\frac{1}{2^{k-1}} \sum_{m=1}^{(k+1) / 2}\left(\begin{array}{c}
k \\
2 m-1
\end{array}\right) n^{k-2 m+1}\left(n^{2}-4\right)^{m-1}
$$

so that $[k]$ is a polynomial in $n$. The polynomials

$$
n^{k}=\left(q+q^{-1}\right)^{k} \quad \text { and } \quad\{k\}=q^{k}+q^{-k} \quad \text { and } \quad[k]=\frac{q^{k}-q^{-k}}{q-q^{-1}}
$$

all form bases of the ring $\mathbb{C}\left[\left(q+q^{-1}\right)\right]$. The transition matrix $B$ between the $[k]$ and the $\{k\}$ is triangular (with 1 s on the diagonal) and the transition matrix $C$ between the $n^{k}$ and the $\{k\}$ is also triangular (the non zero entries are binomial coefficients). Hence, the transition matrix $B C^{-1}$ between $[k]$ and $n^{k}$ has integer entries and so $[k]$ is, in fact, a polynomial in $n$ with integer coefficients.

\subsection{Affine Temperley-Lieb algebras}

The affine Temperley-Lieb algebra $T_{k}^{a}$ is the diagram algebra generated by

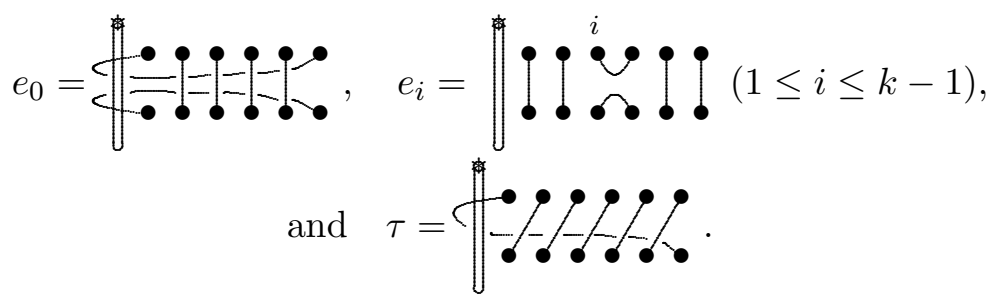

The generators of $T_{k}^{a}$ satisfy $e_{i}^{2}=n e_{i}, e_{i} e_{i \pm 1} e_{i}=e_{i}, \tau e_{i} \tau^{-1}=e_{i+1}$ (where the indices are taken $\bmod k$ ) and

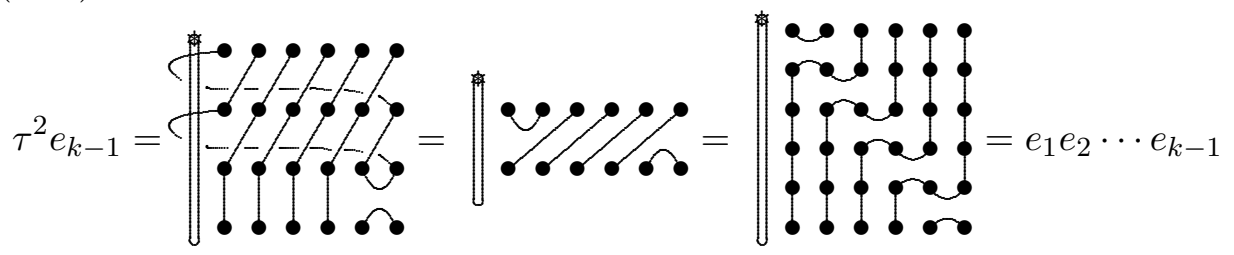

(see $[\mathrm{GL} 2,4.15(\mathrm{iv})]$ ). In $T_{k}^{a}$, we let $X^{\varepsilon_{1}}=T_{1}^{-1} T_{2}^{-1} \cdots T_{k-1}^{-1} \tau^{-1}$ (see Remark $2.1)$. 
Graham and Lehrer [GL2, §4.3] define four slightly different affine Temperley-Lieb algebras, the diagram algebra $T_{k}^{a}$ and the algebras defined as follows:

Type $G L_{k}: \quad \widehat{T L}_{k}^{a}$ is $\tilde{H}_{k}$ with the relation (2.10),

Type $S L_{k}: \quad T L_{k}^{a}$ is $\tilde{H}_{Q}$ with the relation $(2.10)$,

Type $P G L_{k}: \quad \widetilde{T L}_{k}^{a}$ is $\tilde{H}_{P}$ with the relation $(2.10)$.

For each invertible element $\alpha$ in the base ring there is a surjective homomorphism

$$
\begin{aligned}
\tilde{H}_{k} & \longmapsto \widehat{T L}_{k}^{a} \longrightarrow T_{k}^{a} \\
\tau & \longmapsto \tau \\
T_{i} \longmapsto q-e_{i} & \longmapsto q-e_{i}
\end{aligned}
$$

and every irreducible representation of $\widehat{T L}_{k}^{a}$ factors through one of these homomorphisms (see [GL2, Prop. 4.14(v)]). In Proposition 3.2 we shall see that these homomorphisms arise naturally in the Schur-Weyl duality setting.

Remark 2.6. Following [GL1, Cor. (5.11) and Thm. (6.1)], for any irreducible $\widehat{T L}_{k}^{a}$-module there is a constant $x$ such that

$$
e_{1} X^{-\varepsilon_{1}} e_{1}=x e_{1}
$$

In other words, the representation theory (at least the full information of the cell modules, see [GL1, §6], and irreducible modules) is completely contained in the algebras obtained by adding the relation (2.14) to $\widehat{T L}_{k}^{a}$.

\subsection{A commuting family in the affine Temperley-Lieb algebra}

View the elements $X^{-\varepsilon_{i}}$ in the affine Temperley-Lieb algebra $\widehat{T L}_{k}^{a}$ via the surjective algebra homomorphism of (2.13). Define

$$
\begin{aligned}
\left(q-q^{-1}\right) m_{1} & =q^{-1} X^{-\varepsilon_{1}} \quad \text { and } \\
\left(q-q^{-1}\right) m_{i} & =q^{i-2}\left(X^{-\varepsilon_{i}}-q^{-2} X^{-\varepsilon_{i-1}}\right),
\end{aligned}
$$

for $i=2,3, \ldots, k$. Since $X^{-\varepsilon_{i}} X^{-\varepsilon_{j}}=X^{-\varepsilon_{j}} X^{-\varepsilon_{i}}$ for all $1 \leq i, j \leq k$, and the $m_{i}$ are linear combinations of the $X^{-\varepsilon_{i}}$,

$$
m_{i} m_{j}=m_{j} m_{i} \text { in } \widehat{T L}_{k}^{a}, \quad \text { for all } 1 \leq i, j \leq k .
$$


Proposition 2.7. For $1 \leq i \leq k$,

(a) $X^{-\varepsilon_{i}}=q^{-(i-2)}\left(q-q^{-1}\right)\left(m_{i}+q^{-1} m_{i-1}+q^{-2} m_{i-2}+\cdots+q^{-(i-1)} m_{1}\right)$,

(b) $X^{-\varepsilon_{1}}+\cdots+X^{-\varepsilon_{i}}=q^{-(i-2)}\left(q-q^{-1}\right)\left(m_{i}+[2] m_{i-1}+\cdots+[i] m_{1}\right)$.

Proof. Rewrite (2.15) as

$$
X^{-\varepsilon_{i}}=q^{-(i-2)}\left(q-q^{-1}\right) m_{i}+q^{-2} X^{-\varepsilon_{i-1}}
$$

and use induction,

$$
\begin{aligned}
X^{-\varepsilon_{i}}= & q^{-(i-2)}\left(q-q^{-1}\right) m_{i} \\
& +q^{-2}\left(q^{-(i-1-2)}\left(q-q^{-1}\right)\left(m_{i-1}+q^{-1} m_{i-2}+\cdots+q^{-(i-2)} m_{1}\right)\right),
\end{aligned}
$$

to obtain the formula for $X^{-\varepsilon_{i}}$ in (a). Summing the formula in (a) over $i$ gives

$$
\begin{aligned}
\sum_{j=1}^{i} X^{-\varepsilon_{j}} & =\sum_{j=1}^{i}\left(q^{-(j-2)}\left(q-q^{-1}\right) \sum_{\ell=0}^{j-2} q^{-\ell} m_{j-\ell}\right) \\
& =q^{-(i-2)}\left(q-q^{-1}\right) \sum_{j=1}^{i} \sum_{\ell=0}^{j-1} q^{i-j-\ell} m_{j-\ell}
\end{aligned}
$$

and, thus, formula (b) follows from

$$
\begin{aligned}
\sum_{j=1}^{i} \sum_{\ell=0}^{j-1} q^{i-j-\ell} m_{j-\ell} & =\sum_{j=1}^{i} \sum_{r=1}^{j} q^{i-j-(j-r)} m_{r}=\sum_{r=1}^{i} \sum_{j=r}^{i} q^{i+r-2 j} m_{r} \\
& =\sum_{r=1}^{i}[i-r+1] m_{r} .
\end{aligned}
$$

The following Lemma is a transfer of the recursion $X^{\varepsilon_{i}}=T_{i-1} X^{\varepsilon_{i-1}} T_{i-1}$ to the $m_{i}$. The following are the base cases of Lemma 2.8.

$$
m_{1}=\frac{q^{-1}}{q-q^{-1}} X^{-\varepsilon_{1}} \quad \text { and } \quad m_{2}=\frac{x}{q-q^{-1}} e_{1}-\left(e_{1} m_{1}+m_{1} e_{1}\right)
$$


Lemma 2.8. Following Remark 2.6, assume that $e_{1} X^{-\varepsilon_{1}} e_{1}=x e_{1}$ for some constant $x$. For $2 \leq i \leq k$, $m_{i}=\frac{q^{i-2} x}{q-q^{-1}} e_{i-1}-\left(e_{i-1} m_{i-1}+m_{i-1} e_{i-1}\right)-\sum_{\ell=1}^{i-2}([i-\ell]-[i-\ell-2]) m_{\ell} e_{i-1}$.

Proof. From (2.3) and (2.9) we have $X^{-\varepsilon_{i}}=\left(q^{-1}-e_{i-1}\right) X^{-\varepsilon_{i-1}}\left(q^{-1}-\right.$ $\left.e_{i-1}\right)$. Substituting this into the definition of $m_{i}$ gives

$$
\begin{aligned}
\left(q-q^{-1}\right) m_{i} & =q^{i-2}\left(X^{-\varepsilon_{i}}-q^{-2} X^{-\varepsilon_{i-1}}\right) \\
& =q^{i-2}\left(q^{-1}-e_{i-1}\right) X^{-\varepsilon_{i-1}}\left(q^{-1}-e_{i-1}\right)-q^{i-4} X^{-\varepsilon_{i-1}} \\
& =q^{i-2} e_{i-1} X^{-\varepsilon_{i-1}} e_{i-1}-q^{i-3}\left(e_{i-1} X^{-\varepsilon_{i-1}}+X^{-\varepsilon_{i-1}} e_{i-1}\right) .
\end{aligned}
$$

Use Proposition 2.7 (a) to substitute for $X^{-\varepsilon_{i-1}}$,

$$
\begin{aligned}
(q- & \left.q^{-1}\right) m_{i} \\
= & \left(q-q^{-1}\right) q^{-(m+i-3)}\left(q^{i-2} e_{i-1} m_{i-1} e_{i-1}-q^{i-3}\left(e_{i-1} m_{i-1}+m_{i-1} e_{i-1}\right)\right) \\
& +\left(q-q^{-1}\right) q^{-(i-3)}\left(q^{-1} m_{i-2}+\cdots+q^{-(i-2)} m_{1}\right)\left(q^{i-2} e_{i-1}^{2}-2 q^{i-3} e_{i-1}\right) \\
= & \left(q-q^{-1}\right)\left(q e_{i-1} m_{i-1} e_{i-1}-\left(e_{i-1} m_{i-1}+m_{i-1} e_{i-1}\right)\right) \\
& +\left(q-q^{-1}\right)\left(m_{i-2}+\cdots+q^{-(i-3)} m_{1}\right)\left(q+q^{-1}-2 q^{-1}\right) e_{i-1} \\
= & \left(q-q^{-1}\right)\left(\begin{array}{c}
q e_{i-1} m_{i-1} e_{i-1}-\left(e_{i-1} m_{i-1}+m_{i-1} e_{i-1}\right) \\
+\left(q-q^{-1}\right)\left(m_{i-2}+\cdots+q^{-(i-3)} m_{1}\right) e_{i-1}
\end{array}\right),
\end{aligned}
$$

which gives

$$
\begin{aligned}
& m_{i}=q e_{i-1} m_{i-1} e_{i-1}-\left(e_{i-1} m_{i-1}+m_{i-1} e_{i-1}\right) \\
& \quad+\left(q-q^{-1}\right)\left(m_{i-2}+q^{-1} m_{i-3}+q^{-2} m_{i-4}+\cdots+q^{-(i-3)} m_{1}\right) e_{i-1}
\end{aligned}
$$

Using induction, substitute for the first $m_{i-1}$ in this equation to get

$$
\begin{aligned}
m_{i}= & -\left(e_{i-1} m_{i-1}+m_{i-1} e_{i-1}\right)+\left(q-q^{-1}\right) \sum_{\ell=1}^{i-2} q^{-(i-2-\ell)} m_{\ell} e_{i-1} \\
& +q\left(\frac{q^{i-3} x}{q-q^{-1}} e_{i-1}-2 m_{i-2} e_{i-1}-\sum_{\ell=1}^{i-3}([i-\ell-1]-[i-\ell-3]) m_{\ell} e_{i-1}\right) \\
= & \frac{q^{i-2} x}{q-q^{-1}} e_{i-1}-\left(e_{i-1} m_{i-1}+m_{i-1} e_{i-1}\right)-\sum_{\ell=2}^{i-2}([i-\ell]-[i-\ell-2]) m_{\ell} e_{i-1} .
\end{aligned}
$$




\subsection{Diagram representation of Murphy elements}

Label the vertices from left to right in the top row of a diagram $d \in T_{k}$ with $1,2, \ldots, k$, and label the corresponding vertices in the bottom row with $1^{\prime}, 2^{\prime}, \ldots, k^{\prime}$. The cycle type of a diagram $d \in T_{k}$ is the set partition $\tau(d)$ of $\{1,2, \ldots, k\}$ obtained from $d$ by setting $1=1^{\prime}, 2=2^{\prime}, \ldots, k=k^{\prime}$. If $\tau(d)$ is a set partition of the form $\left\{\left\{1,2, \ldots, \gamma_{1}\right\},\left\{\gamma_{1}+1, \mu_{1}+2, \ldots, \gamma_{1}+\gamma+\right.\right.$ $\left.2\}, \ldots,\left\{\gamma_{1}+\cdots+\gamma_{\ell-1}+1, \ldots, k\right\}\right\}$, where $\left(\gamma_{1}, \ldots, \gamma_{\ell}\right)$ is a composition of $k$, then we simplify notation by writing $\tau(d)=\left(\gamma_{1}, \ldots, \gamma_{\ell}\right)$. For example

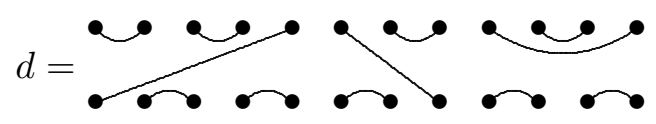

has $\tau(d)=(5,3,4)$.

There are diagrams whose cycle type cannot be written as a composition (for example $d=\underset{0}{\text { o. }}$ has cycle type $\{\{1,4\},\{2,3\}\})$ but all of the diagrams needed here have cycle types that are compositions.

If $\gamma=\left(\gamma_{1}, \ldots, \gamma_{\ell}\right)$ is a composition of $k$ define

$$
d_{\gamma}=\sum_{\tau(d)=\gamma} d
$$

as the sum of the Temperley-Lieb diagrams on $k$ dots with cycle type $\gamma$. Define $d_{\gamma}^{*}$ be the sum of diagrams obtained from the summands of $d_{\gamma}$ by wrapping the first edge in each row around the pole, with the orientation coming from $X^{-\varepsilon_{1}}$ as shown in the examples below. When the first edge in the top row connects to the first vertex in the bottom row only one new diagram is produced, otherwise there are two. For example, in $\widehat{T L}_{4}^{a}$,

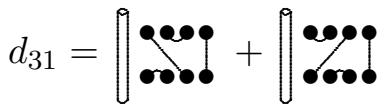

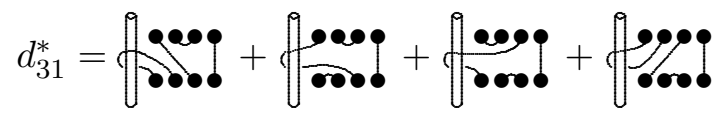

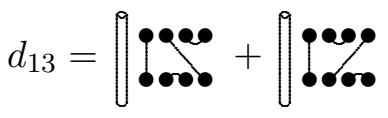

$$
\begin{aligned}
& d_{13}^{*}=\text { foroce }+ \text { foocos }
\end{aligned}
$$

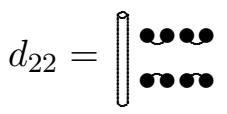

$$
\begin{aligned}
& d_{22}^{*}=\text { fooce }+ \text { fopec }
\end{aligned}
$$

View $d_{\gamma}$ and $d_{\gamma}^{*}$ as elements of $\widehat{T L}_{k}^{a}$ by setting

$$
d_{\gamma}=d_{\gamma 1^{k-i}}, \quad \text { if } \gamma \text { is a composition of } i \text { with } i<k \text {. }
$$


With this notation, expanding the first few $m_{i}$ in terms of diagrams gives

$$
\begin{aligned}
\left(q-q^{-1}\right) m_{1}= & q^{-1} d_{1}^{*}, \quad\left(q-q^{-1}\right) m_{2}=x d_{2}-q^{-1} d_{2}^{*}, \\
\left(q-q^{-1}\right) m_{3}= & q x d_{1,2}-q^{-1}[2] d_{1,2}^{*}-x d_{3}+q^{-1} d_{3}^{*}, \\
\left(q-q^{-1}\right) m_{4}= & q^{2} x d_{1^{2}, 2}-q^{-1}([3]-[1]) d_{1^{2}, 2}^{*}-x[2] d_{2,2}+q^{-1}[2] d_{2,2}^{*} \\
& -q x d_{1,3}+q^{-1}[2] d_{1,3}^{*}+x d_{4}-q^{-1} d_{4}^{*}, \\
\left(q-q^{-1}\right) m_{5}= & q^{3} x d_{1^{3}, 2}-q^{-1}([4]-[2]) d_{1^{3}, 2}^{*}-q^{2} x d_{1^{2}, 3}+q^{-1}([3]-[1]) d_{1^{2}, 3}^{*} \\
& +q x d_{1,4}-q^{-1}[2] d_{1,4}^{*}-q x[2] d_{1,2,2}+q^{-1}[2]^{2} d_{1,2,2}^{*}+x[2] d_{2,3} \\
& -q^{-1}[2] d_{2,3}^{*}-x([3]-[1]) d_{2,1,2}+q^{-1}([3]-[1]) d_{2,1,2}^{*} \\
& +x[2] d_{3,2}-q^{-1}[2] d_{3,2}^{*}-x d_{5}+q^{-1} d_{5}^{*},
\end{aligned}
$$

where, as in Lemma 2.8, $x$ is the constant defined by the equation $e_{1} X^{-\varepsilon_{1}} e_{1}=x e_{1}$.

ThEOREM 2.9. Following Remark 2.6, assume that $e_{1} X^{-\varepsilon_{1}} e_{1}=x e_{1}$ for some constant $x$. Then $\left(q-q^{-1}\right) m_{1}=q^{-1} d_{1}^{*},\left(q-q^{-1}\right) m_{2}=x d_{2}-q^{-1} d_{2}^{*}$ and, for $i \geq 2$,

$$
m_{i}=\sum_{\text {compositions } \gamma}\left(m_{i}\right)_{\gamma} d_{\gamma}+\left(m_{i}\right)_{\gamma}^{*} d_{\gamma}^{*}
$$

where the sum is over all compositions $\gamma=1^{b_{1}} r_{1} 1^{b_{2}} r_{2} \cdots 1^{b_{\ell}} r_{\ell}$ of $i$ with $r_{\ell}>1$, and

$$
\begin{aligned}
& \left(m_{i}\right)_{\gamma}=(-1)^{|\gamma|-\ell(\gamma)-1} \frac{q^{b_{1}} x}{q-q^{-1}} \prod_{j>1}\left(\left[b_{j}+2\right]-\left[b_{j}\right]\right), \quad \text { and } \\
& \left(m_{i}\right)_{\gamma}^{*}=(-1)^{|\gamma|-\ell(\gamma)} \frac{q^{-1}}{q-q^{-1}}\left(\left[b_{1}+1\right]-\left[b_{1}-1\right]\right) \prod_{j>1}\left(\left[b_{j}+2\right]-\left[b_{j}\right]\right),
\end{aligned}
$$

with $\ell(\gamma)=\ell+b_{1}+\cdots+b_{\ell}$.

Proof. From our computations above, $m_{1}=A d_{1}^{*}$ and $m_{2}=B d_{2}-A d_{2}^{*}$, where

$$
A=\frac{q^{-1}}{q-q^{-1}} \quad \text { and } \quad B=\frac{x}{q-q^{-1}} .
$$


Let $m_{1}=A d_{1}^{*}$. For $i>2$ the recursion in Lemma 2.8 gives

$$
\begin{aligned}
m_{i}= & q^{i-2} B e_{i-1}-\left(e_{i-1} m_{i-1}+m_{i-1} e_{i-1}\right)-\sum_{\ell=1}^{i-2}([i-\ell]-[i-\ell-2]) m_{\ell} e_{i-1} \\
= & q^{i-2} B d_{1^{i-2}, 2}-\left(\sum_{\gamma=\gamma^{\prime} r}\left(\left(m_{i-1}\right)_{\gamma^{\prime} r} d_{\gamma^{\prime}, r+1}+\left(m_{i-1}\right)_{\gamma^{\prime} r}^{*} d_{\gamma^{\prime}, r+1}^{*}\right)\right) \\
& -([i-1]-[i-3]) A d_{1^{i-2}, 2}^{*} \\
& +\sum_{\ell=2}^{i-2}-([i-\ell]-[i-\ell-2]) \sum_{\gamma^{\prime}}\left(\left(m_{\ell}\right)_{\gamma^{\prime}} d_{\gamma^{\prime} 1^{i-2-\ell_{2}}}+\left(m_{\ell}\right)_{\gamma^{\prime}}^{*} d_{\gamma^{\prime} 1^{i-2-\ell_{2}}}^{*}\right) .
\end{aligned}
$$

So if $d$ has cycle type $\gamma=1^{b_{1}} r_{1} 1^{b_{2}} r_{2} \cdots 1^{b_{\ell}} r_{\ell}$ with $r_{\ell}>0$, then

(a) Each part of size $r(r>1)$ contributes $(-1)^{r-1}$ to the coefficient. Thus, there is a total contribution of $(-1)^{|\gamma|-\ell(\gamma)}$ from these parts.

(b) Each inner $1^{b}(b \geq 0)$ contributes a factor of $[b+2]-[b]$ to the coefficient.

(c) The first $1^{b}(b>0)$ contributes $a-q^{b} B$ in a nonstarred class,

$\left(c^{\prime}\right)$ The first $1^{b}(b=0)$ contributes $a-B$ in a nonstarred class, which is the same as case $(c)$ with $b=0$.

(d) The first $1^{b}(b>0)$ contributes a $([b+1]-[b-1]) A$ in a starred class.

$\left(d^{\prime}\right)$ The first $1^{b}(b=0)$ contributes an $A$ in a starred class, which is the same as case $(d)$ with $b=0$ assuming $[-1]=0$.

Remark 2.10. To view $m_{1}, \ldots, m_{k}$ in the (nonaffine) Temperley-Lieb algebra $T L_{k}(n)$ (via $(2.11)$ ) let $X^{-\varepsilon_{1}}=1$ so that $x=q+q^{-1}$. If $b_{1}>1$ then $d_{\gamma}^{*}=d_{\gamma}$ and if $b_{1}=0$ then $d_{\gamma}^{*}=2 d_{\gamma}$. In both cases the coefficients in Theorem 2.9 specialize to

$$
\left(m_{i}\right)_{\gamma}+\left(m_{i}\right)_{\gamma}^{*}=(-1)^{|\gamma|-\ell(\gamma)-1}\left[b_{1}+1\right] \prod_{b_{j} \geq 0, j>1}\left(\left[b_{j}+2\right]-\left[b_{j}\right]\right)
$$

and

$$
m_{i}=\sum_{\gamma}\left(\left(m_{i}\right)_{\gamma}+\left(m_{i}\right)_{\gamma}^{*}\right) d_{\gamma}
$$


where the sum is over compositions $\gamma=1^{b_{1}} r_{1} 1^{b_{2}} r_{2} \cdots 1^{b_{\ell}} r_{\ell}$ of $i$ with $r_{\ell}>1$. The first few examples are

$$
\begin{aligned}
& m_{1}= \frac{q^{-1}}{q-q^{-1}}=\frac{q^{-1}}{q-q^{-1}} d_{1}, \quad m_{2}=e_{2}=d_{2}, \quad m_{3}=[2] d_{12}-d_{3}, \\
& m_{4}=[3] d_{1^{2}, 2}-[2] d_{2,2}-[2] d_{1,3}+d_{4}, \\
& m_{5}=[4] d_{1^{3}, 2}-[3] d_{1^{2}, 3}+[2] d_{1,4}-[2]^{2} d_{1,2,2}+[2] d_{2,3}-([3]-[1]) d_{2,1,2} \\
&+[2] d_{3,2}-d_{5} .
\end{aligned}
$$

\section{$\S 3$. Schur functors}

\section{1. $R$-matrices and quantum Casimir elements}

Let $U_{h} \mathfrak{g}$ be the Drinfeld-Jimbo quantum group corresponding to a finite dimensional complex semisimple Lie algebra $\mathfrak{g}$. We shall use the notations and conventions for $U_{h} \mathfrak{g}$ as in [LR] and [OR]. There is an invertible element $\mathcal{R}=\sum a_{i} \otimes b_{i}$ in (a suitable completion of) $U_{h} \mathfrak{g} \otimes U_{h} \mathfrak{g}$ such that, for two $U_{h} \mathfrak{g}$ modules $M$ and $N$, the map

$$
\begin{aligned}
\check{R}_{M N}: M \otimes N & \longrightarrow N \otimes M \\
m \otimes n & \longmapsto \sum b_{i} n \otimes a_{i} m
\end{aligned}
$$

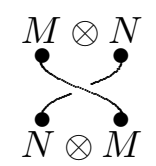

is a $U_{h} \mathfrak{g}$ module isomorphism. In order to be consistent with the graphical calculus these operators should be written on the right. The element $\mathcal{R}$ satisfies "quasitriangularity relations" (see [LR, (2.1)-(2.3)]) which imply that, for $U_{h} \mathfrak{g}$ modules $M, N, P$ and a $U_{h} \mathfrak{g}$ module isomorphism $\tau_{M}: M \rightarrow$ $M$,

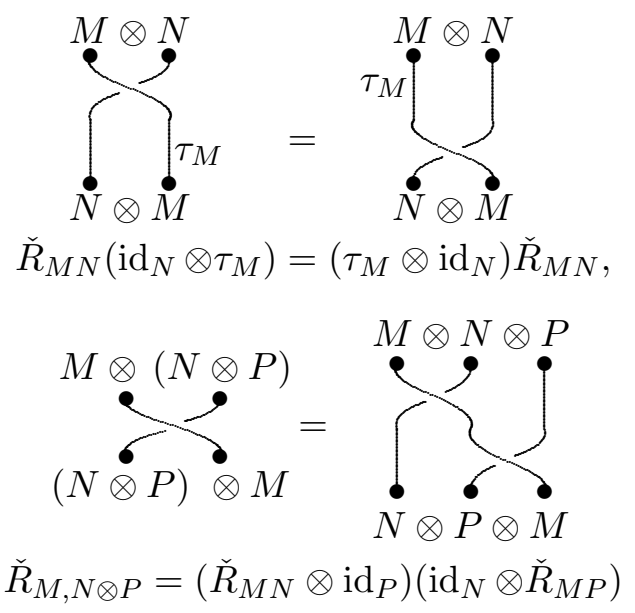




$$
P \otimes(M \otimes N)=\left(\check{R}_{M \otimes N, P}=\left(\operatorname{id}_{M} \otimes \check{R}_{N P}\right)\left(\check{R}_{M P} \otimes \operatorname{id}_{N}\right),\right.
$$

which, together, imply the braid relation

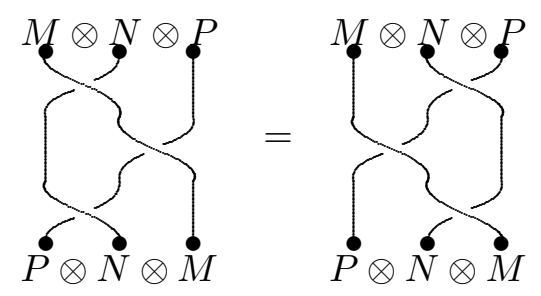

$\left(\check{R}_{M N} \otimes \operatorname{id}_{P}\right)\left(\operatorname{id}_{N} \otimes \check{R}_{M P}\right)\left(\check{R}_{N P} \otimes \operatorname{id}_{M}\right)$

$$
=\left(\operatorname{id}_{M} \otimes \check{R}_{N P}\right)\left(\check{R}_{M P} \otimes \operatorname{id}_{N}\right)\left(\operatorname{id}_{P} \otimes \check{R}_{M N}\right) .
$$

Let $\rho$ be such that $\left\langle\rho, \alpha_{i}\right\rangle=1$ for all simple roots $\alpha_{i}$. As explained in $[\mathrm{LR},(2.14)]$ and $[\mathrm{Dr}]$, there is a quantum Casimir element $e^{-h \rho} u$ in the center of $U_{h} \mathfrak{g}$ and, for a $U_{h} \mathfrak{g}$ module $M$ we define a $U_{h} \mathfrak{g}$ module isomorphism

$$
\begin{aligned}
C_{M}: M & \longrightarrow\left(\begin{array}{c}
M \\
m
\end{array} e^{-h \rho} u\right) m
\end{aligned}
$$

and the elements $C_{M}$ satisfy

$$
C_{M \otimes N}=\left(\check{R}_{M N} \check{R}_{N M}\right)^{-1}\left(C_{M} \otimes C_{N}\right), \quad \text { and } \quad C_{M}=q^{-\langle\lambda, \lambda+2 \rho\rangle} \operatorname{id}_{M}
$$

if $M$ is a $U_{h} \mathfrak{g}$ module generated by a highest weight vector $v^{+}$of weight $\lambda$ (see [LR, Prop. 2.14] or [Dr, Prop. 3.2]). Note that $\langle\lambda, \lambda+2 \rho\rangle=\langle\lambda+\rho, \lambda+$ $\rho\rangle-\langle\rho, \rho\rangle$ are the eigenvalues of the classical Casimir operator [Dx, 7.8.5]. From the relation (3.1) it follows that if $M=L(\mu), N=L(\nu)$ are finite dimensional irreducible $U_{h} \mathfrak{g}$ modules then $\check{R}_{M N} \check{R}_{N M}$ acts on the $\lambda$ isotypic component $L(\lambda)^{\oplus c_{\mu \nu}^{\lambda}}$ of the decomposition

$$
L(\mu) \otimes L(\nu)=\bigoplus_{\lambda} L(\lambda)^{\oplus c_{\mu \nu}^{\lambda}}
$$

by the constant $q^{\langle\lambda, \lambda+2 \rho\rangle-\langle\mu, \mu+2 \rho\rangle-\langle\nu, \nu+2 \rho\rangle}$. 


\subsection{The $\tilde{\mathcal{B}}_{k}$ module $M \otimes V^{\otimes k}$}

Let $U=U_{h} \mathfrak{g}$ be a quantum group and let $M$ and $V$ be $U$-modules such that the operators $\check{R}_{M V}, \check{R}_{V M}$ and $\check{R}_{V V}$ are well defined. Define $\check{R}_{i}$, $1 \leq i \leq k-1$, and $\check{R}_{0}^{2}$ in $\operatorname{End}_{U}\left(M \otimes V^{\otimes k}\right)$ by

$$
\begin{array}{ll}
\check{R}_{i} & =\operatorname{id}_{M} \otimes \operatorname{id}_{V}^{\otimes(i-1)} \otimes \check{R}_{V V} \otimes \operatorname{id}_{V}^{\otimes(k-i-1)} \text { and } \\
\check{R}_{0}^{2} & =\left(\check{R}_{M V} \check{R}_{V M}\right) \otimes \operatorname{id}_{V}^{\otimes(k-1)} .
\end{array}
$$

Then the braid relations

$$
\left.\left.\check{R}_{i} \check{R}_{i+1} \check{R}_{i}=\right\}=\right\}=\check{R}_{i+1} \check{R}_{i} \check{R}_{i+1}
$$

and

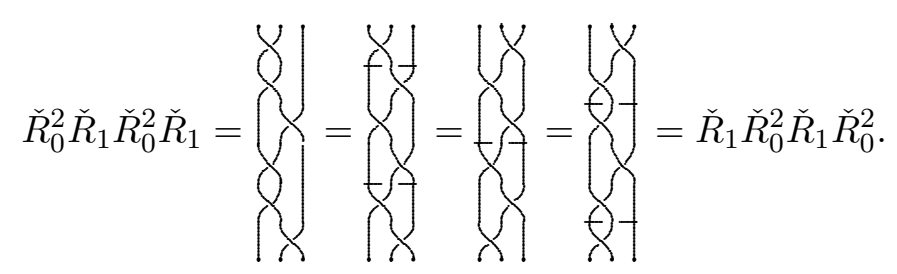

imply that there is a well defined map

$$
\begin{aligned}
& \Phi: \quad \tilde{\mathcal{B}}_{k} \longrightarrow \operatorname{End}_{U}\left(M \otimes V^{\otimes k}\right) \\
& T_{i} \longmapsto \quad \check{R}_{i}, \quad 1 \leq i \leq k-1 \text {, } \\
& X^{\varepsilon_{1}} \longmapsto \quad \check{R}_{0}^{2},
\end{aligned}
$$

which makes $M \otimes V^{\otimes k}$ into a right $\tilde{\mathcal{B}}_{k}$ module. By (3.1) and the fact that

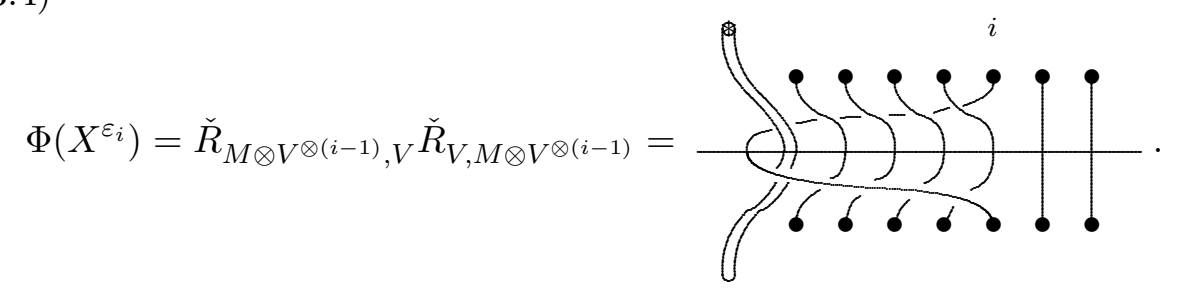

the eigenvalues of $\Phi\left(X^{\varepsilon_{i}}\right)$ are related to the eigenvalues of the Casimir. The Schur functors are the functors

$$
\begin{aligned}
& F_{V}^{\lambda}:\{U \text {-modules }\} \longrightarrow \quad\left\{\tilde{\mathcal{B}}_{k} \text {-modules }\right\} \\
& M \quad \longmapsto \operatorname{Hom}_{U}\left(M(\lambda), M \otimes V^{\otimes k}\right)
\end{aligned}
$$

where $\operatorname{Hom}_{U}\left(M(\lambda), M \otimes V^{\otimes k}\right)$ is the vector space of highest weight vectors of weight $\lambda$ in $M \otimes V^{\otimes k}$. 


\subsection{The quantum group $U_{h} \mathfrak{g l}_{n}$}

Although the Lie algebra $\mathfrak{g l}_{n}$ is reductive, not semisimple, all of the general setup of Sections 3.1 and 3.2 can be applied without change. The simple roots are $\alpha_{i}=\varepsilon_{i}-\varepsilon_{i+1}, 1 \leq i \leq n-1$, and

$$
\rho=(n-1) \varepsilon_{1}+(n-2) \varepsilon_{2}+\cdots+\varepsilon_{n-1} .
$$

The dominant integral weights of $\mathfrak{g l}_{n}$ are

$$
\lambda=\lambda_{1} \varepsilon_{1}+\cdots+\lambda_{n} \varepsilon_{n}, \quad \text { where } \lambda_{1} \geq \lambda_{2} \geq \cdots \geq \lambda_{n} \text {, and } \lambda_{1}, \ldots, \lambda_{n} \in \mathbb{Z},
$$

and these index the simple finite dimensional $U_{h} \mathfrak{g l}_{n}$-modules $L(\lambda)$. A partition with $\leq n$ rows is a dominant integral weight with $\lambda_{n} \geq 0$. If $\lambda_{n}<0$ and $\Delta$ denotes the 1-dimensional "determinant" representation of $U_{h} \mathfrak{g l}_{n}$ then (see [FH, §15.5])

$$
\begin{aligned}
L(\lambda) \cong & \Delta^{\lambda_{n}} \otimes L\left(\lambda+\left(-\lambda_{n}, \ldots,-\lambda_{n}\right)\right) \\
& \text { with } \lambda+\left(-\lambda_{n}, \ldots,-\lambda_{n}\right) \text { a partition. }
\end{aligned}
$$

Identify each partition $\lambda$ with the configuration of boxes which has $\lambda_{i}$ boxes in row $i$. For example,

$$
\lambda=\sqrt{\bigoplus H}=5 \varepsilon_{1}+5 \varepsilon_{2}+3 \varepsilon_{3}+3 \varepsilon_{4}+\varepsilon_{5}+\varepsilon_{6} .
$$

If $\mu$ and $\lambda$ are partitions with $\mu \subseteq \lambda$ (as collections of boxes) then the skew shape $\lambda / \mu$ is the collection of boxes of $\lambda$ that are not in $\mu$. For example, if $\lambda$ is as in (3.8) and

$$
\mu=\bigoplus \text { then } \lambda / \mu=\underset{\theta}{\square} \text {. }
$$

If $b$ is a box in position $(i, j)$ of $\lambda$ the content of $b$ is

(3.9) $c(b)=j-i=$ the diagonal number of $b$, so that

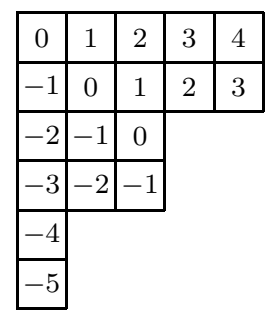


are the contents of the boxes for the partition in (3.8).

If $\nu$ is a partition and

$$
V=L(\square) \text { then } L(\nu) \otimes V=\bigoplus_{\lambda \in \nu^{+}} L(\lambda),
$$

where the sum is over all partitions $\lambda$ with $\leq n$ rows that are obtained by adding a box to $\nu$ [Mac, I App. A (8.4) and I (5.16)], Hence, the $U_{h} \mathfrak{g l}_{n^{-}}$ module decompositions of

$$
L(\mu) \otimes V^{\otimes k}=\bigoplus_{\lambda} L(\lambda) \otimes \tilde{H}_{k}^{\lambda / \mu}, \quad k \in \mathbb{Z}_{\geq 0},
$$

are encoded by the graph $\hat{H}^{/ \mu}$ with

$$
\begin{array}{ll}
\text { vertices on level } k: & \text { \{skew shapes } \lambda / \mu \text { with } k \text { boxes }\} \\
\text { edges: } & \lambda / \mu \longrightarrow \gamma / \mu, \text { if } \gamma \text { is obtained from } \lambda \\
& \text { by adding a box } \\
\text { labels on edges: } & \text { content of the added box. }
\end{array}
$$

For example if $\mu=(3,3,3,2)=\square$, then the first 4 rows of $\hat{H}^{/ \mu}$ are

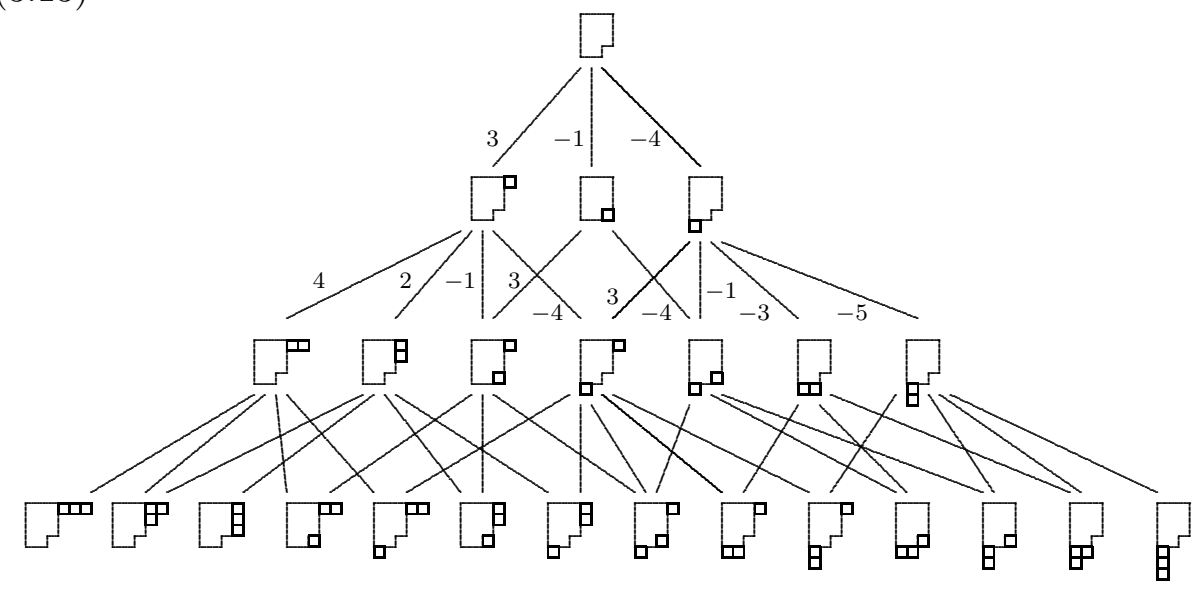

The following result is well known (see [Ji] or [LR, (4.4)]).

Proposition 3.1. If $U=U_{h} \mathfrak{g l}_{n}$ and $V=L\left(\varepsilon_{1}\right)=L(\square)$ is the $n$ dimensional "standard" representation of $\mathfrak{g l}_{n}$ then the map $\Phi$ of (3.3) factors through the surjective homomorphism (2.8) to give a representation of the affine Hecke algebra. 
For a skew shape $\lambda / \mu$ with $k$ boxes identify paths from $\mu$ to $\lambda / \mu$ in $\hat{H}^{/ \mu}$ with standard tableaux of shape $\lambda / \mu$ by filling the boxes, successively, with $1,2, \ldots, k$ as they appear. In the example graph $\hat{H}^{/ \mu}$ above

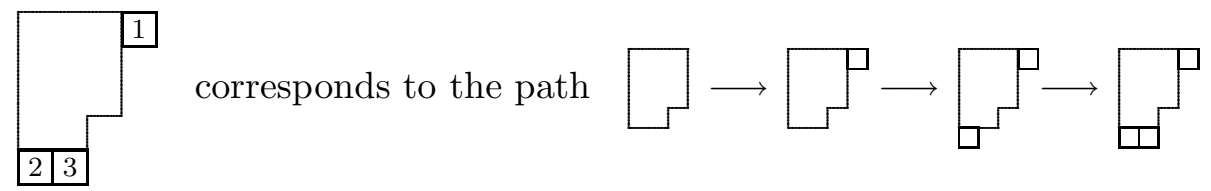

\subsection{The quantum group $U_{h} \mathfrak{g l}_{2}$}

In the case when $n=2, U=U_{h} \mathfrak{g l}_{2}$ and the partitions which appear in (3.11) and in the graph $\hat{H}^{/ \mu}$ all have $\leq 2$ rows. For example if $\mu=(42)=$ $\left\ulcorner\right.$ then the first few rows of $\hat{H}^{/ \mu}$ are

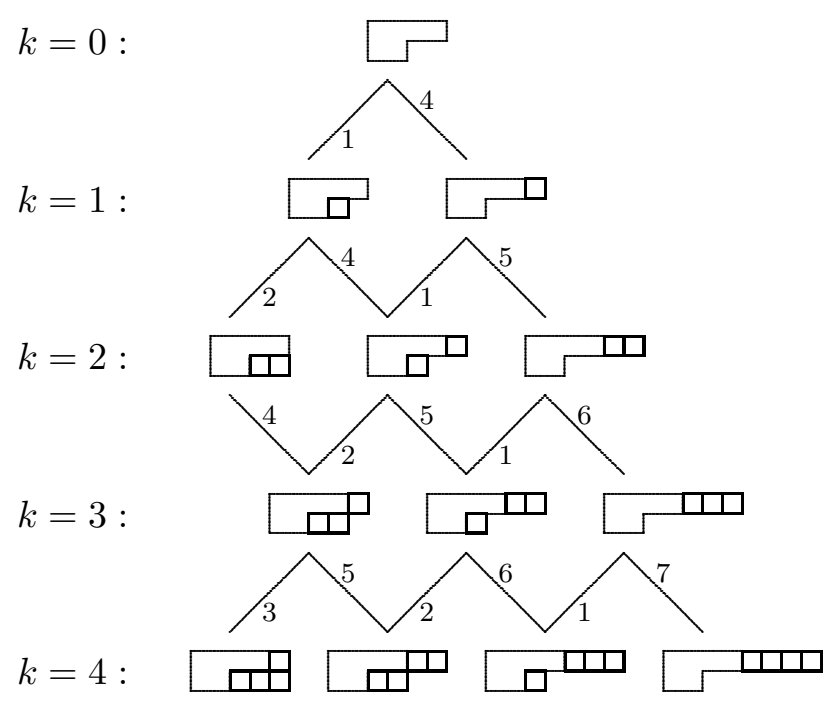

and this is the graph which describes the decompositions in (3.11).

Proposition 3.2. If $U=U_{h} \mathfrak{g l}_{2}, M=L(\mu)$ where $\mu$ is a partition of $m$ with $\leq 2$ rows, and $V=L\left(\varepsilon_{1}\right)=L(\square)$ is the 2 -dimensional "standard" representation of $\mathfrak{g l}_{2}$ then the map $\Phi$ of (3.3) factors through the surjective homomorphism of (2.13) with $\alpha^{2}=-q^{2 m-1}$ to give a representation of the affine Temperley-Lieb algebra $T_{k}^{a}$.

Proof. The proof that the kernel of $\Phi$ contains the element (2.12) is exactly as in the proof of [OR, Thm. 6.1(c)]: The element $e_{1}$ in $T_{2}^{a}$ acts on 
$V^{\otimes 2}$ as $\left(q+q^{-1}\right) \cdot$ pr where pr is the unique $U_{h} \mathfrak{g}$-invariant projection onto $L(\mathrm{~B})$ in $V^{\otimes 2}$. Using $e_{1} T_{1}=-q^{-1} e_{1}$ and the pictorial equalities
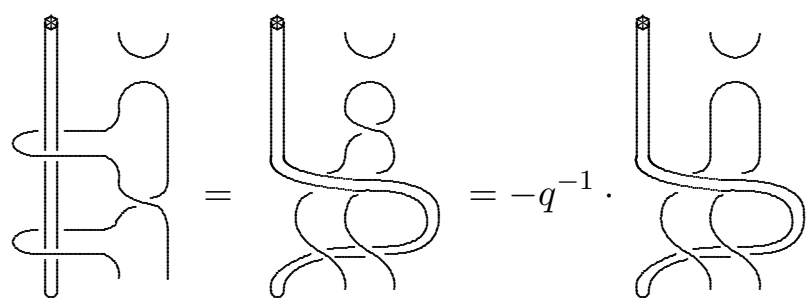

it follows that $\Phi_{2}\left(e_{1} X^{\varepsilon_{1}} T_{1} X^{\varepsilon_{1}}\right)$ acts as $-\left(q+q^{-1}\right) q^{-1} \cdot \check{R}_{L(\text { 日) }, L(\mu)} \check{R}_{L(\mu), L(\text { 日) }}$ $\left(\operatorname{id}_{L(\mu)} \otimes \mathrm{pr}\right)$. By $(3.1)$, this is equal to

$$
\begin{aligned}
& -q^{-1}\left(C_{L(\mu)} \otimes C_{L(\theta)}\right) C_{L(\mu) \otimes L(日)}^{-1} \Phi_{2}\left(\operatorname{id}_{L(\mu)} \otimes e_{1}\right) \\
& \quad=-q^{-1} q^{-\langle\mu, \mu+2 \rho\rangle} q^{-\left\langle\varepsilon_{1}+\varepsilon_{2}, \varepsilon_{1}+\varepsilon_{2}+2 \rho\right\rangle} C_{L\left(\mu+\varepsilon_{1}+\varepsilon_{2}\right)}^{-1} \Phi_{2}\left(\operatorname{id}_{L(\mu)} \otimes e_{1}\right),
\end{aligned}
$$

and the coefficient $-q^{-1} q^{-\langle\mu, \mu+2 \rho\rangle} q^{-\left\langle\varepsilon_{1}+\varepsilon_{2}, \varepsilon_{1}+\varepsilon_{2}+2 \rho\right\rangle} C_{L\left(\mu+\varepsilon_{1}+\varepsilon_{2}\right)}^{-1}$ simplifies to

$$
\begin{aligned}
& -q^{-1} q^{-\langle\mu, \mu+2 \rho\rangle} q^{-\left\langle\varepsilon_{1}+\varepsilon_{2}, \varepsilon_{1}+\varepsilon_{2}+2 \rho\right\rangle} q^{\left\langle\mu+\varepsilon_{1}+\varepsilon_{2}, \mu+\varepsilon_{1}+\varepsilon_{2}+2 \rho\right\rangle} \\
& =-q^{-1} q^{2\left(\mu_{1}+\mu_{2}\right)}=-q^{2 m-1}
\end{aligned}
$$

where $m=\mu_{1}+\mu_{2}=|\mu|$.

\subsection{The quantum group $U_{h} \mathfrak{s l}_{2}$}

The restriction of an irreducible representation $L(\lambda)$ of $U_{h} \mathfrak{g l}_{n}$ to $U_{h} \mathfrak{s l}_{n}$ is irreducible and all irreducible representations of $U_{h} \mathfrak{s l}_{n}$ are obtained in this fashion. Since the "determinant" representation is trivial as an $U_{h} \mathfrak{s l}_{n}$ module it follows from (3.7) that the irreducible representations $L_{\mathfrak{s l}_{n}}(\lambda)$ of $U_{h} \mathfrak{s l}_{n}$ are indexed by partitions $\lambda=\left(\lambda_{1}, \ldots, \lambda_{n}\right)$ with $\lambda_{n}=0$. Hence, the graph which describes the $U_{h} \mathfrak{s l}_{2}$-module decompositions of

$$
L(\mu) \otimes V^{\otimes k}=\bigoplus_{\lambda} L(\lambda) \otimes \tilde{T}_{k}^{\lambda / \mu}, \quad k \in \mathbb{Z}_{\geq 0},
$$

is exactly the same as the graph for $U_{h} \mathfrak{g l}_{2}$ except with all columns of length 2 removed from the partitions. More precisely, the decompositions are encoded by the graph $\hat{T}^{/ \mu}$ with

vertices on level $k: \quad\left\{\mu_{1}-\mu_{2}+k, \mu_{1}-\mu_{2}+k-2, \ldots, \mu_{1}-\mu_{2}-k\right\} \cap \mathbb{Z}_{\geq 0}$

edges: $\quad \ell \longrightarrow \ell \pm 1$. 
For example if $m=7$ and $\mu_{1}-\mu_{2}=3$ then the first few rows of $\hat{T}^{/ \mu}$ are

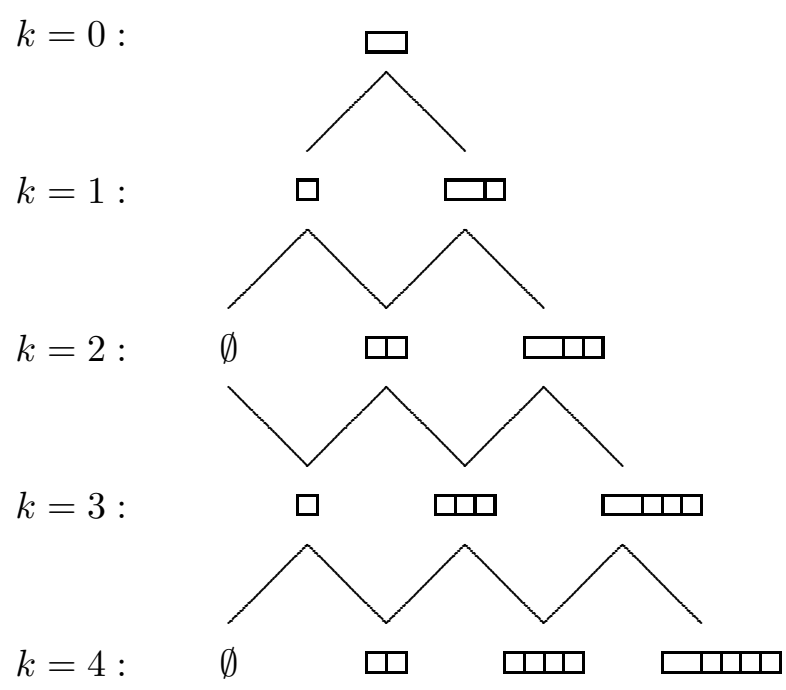

Paths in (3.17) correspond to paths in (3.14) which correspond to standard tableaux $T$ of shape $\lambda / \mu$.

\section{§4. Eigenvalues}

\subsection{Eigenvalues of the $X^{\varepsilon_{i}}$ in the affine Hecke algebra}

Recall, from (2.8), that the affine Hecke algebra $\tilde{H}_{k}$ is the quotient of the group algebra of the affine braid group $\mathbb{C} \tilde{B}_{k}$ by the relations

$$
T_{i}^{2}=\left(q-q^{-1}\right) T_{i}+1
$$

As observed in Proposition 3.2 the map $\Phi$ in (3.3) makes the module $L(\mu) \otimes$ $V^{\otimes k}$ in (3.11) into an $\tilde{H}_{k}$ module. Thus the vector spaces $\tilde{H}_{k}^{\lambda / \mu}$ in $(3.11)$ are the $\tilde{H}_{k}$-modules given by

$$
\tilde{H}_{k}^{\lambda / \mu}=F_{V}^{\lambda}(L(\mu)), \quad \text { where } F_{V}^{\lambda} \text { are the Schur functors of (3.5). }
$$

The following theorem is well known (see, for example, [Ch]).

TheOREM 4.1. (a) The $X^{\varepsilon_{i}}, 1 \leq i \leq k$, mutually commute in the affine Hecke algebra $\tilde{H}_{k}$. 
(b) The eigenvalues of $X^{\varepsilon_{i}}$ are given by the graph $\hat{H}^{/ \mu}$ of (3.13) in the sense that if

$$
\begin{aligned}
\hat{H}_{k}^{/ \mu} & =\{\text { skew shapes } \lambda / \mu \text { with } k \text { boxes }\} \quad \text { and } \\
\hat{H}_{k}^{\lambda / \mu} & =\{\text { standard tableaux } T \text { of shape } \lambda / \mu\}
\end{aligned}
$$

for $\lambda / \mu \in \hat{H}_{k}^{/ \mu}$, then

$\hat{H}_{k}^{/ \mu}$ is an index set for the simple $\tilde{H}_{k}$ modules $\tilde{H}_{k}^{\lambda / \mu}$

appearing in $L(\mu) \otimes V^{\otimes k}$,

and

$$
\tilde{H}_{k}^{\lambda / \mu} \text { has a basis }\left\{v_{T} \mid T \in \hat{H}_{k}^{\lambda / \mu}\right\} \text { with } X^{\varepsilon_{i}} v_{T}=q^{2 c(T(i))} v_{T},
$$

where $c(T(i))$ is the content of box $i$ of $T$.

(c) $\kappa=X^{\varepsilon_{1}} \cdots X^{\varepsilon_{k}}$ is a central element of $\tilde{H}_{k}$ and

$$
\kappa \text { acts on } \tilde{H}_{k}^{\lambda / \mu} \text { by the constant } q^{2 \sum_{b \in \lambda / \mu} c(b)} \text {. }
$$

Proof. (a) is a restatement of (2.4). (b) Since the $\tilde{H}_{k}$ action and the $U_{h} \mathfrak{g l}_{n}$ action commute on $L(\mu) \otimes V^{\otimes k}$ it follows that the decomposition in (3.11) is a decomposition as $\left(U_{h} \mathfrak{g l}_{n}, \tilde{H}_{k}\right)$ bimodules, where the $\tilde{H}_{k}^{\lambda / \mu}$ are some $\tilde{H}_{k}$-modules. Comparing the $L(\lambda)$ components on each side of

$$
\begin{aligned}
\bigoplus_{\lambda} L(\lambda) \otimes \tilde{H}_{\ell}^{\lambda / \mu} & \cong L(\mu) \otimes V^{\otimes \ell}=L(\mu) \otimes V^{\otimes(\ell-1)} \otimes V \\
& \cong\left(\bigoplus_{\nu} L(\nu) \otimes \tilde{H}_{\ell-1}^{\nu / \mu}\right) \otimes V \\
& \cong \bigoplus_{\lambda} \bigoplus_{\lambda / \nu=\square} L(\lambda) \otimes \tilde{H}_{\ell-1}^{\nu / \mu} \\
& \cong \bigoplus_{\lambda}\left(L(\lambda) \otimes\left(\bigoplus_{\lambda / \nu=\square} \tilde{H}_{\ell-1}^{\nu / \mu}\right)\right)
\end{aligned}
$$

gives

$$
\tilde{H}_{\ell}^{\lambda / \mu} \cong \bigoplus_{\lambda / \nu=\square} \tilde{H}_{\ell-1}^{\nu / \mu}
$$


for any $\ell \in \mathbb{Z}_{\geq 0}$ and skew shape $\lambda / \mu$ with $\ell$ boxes. Iterate (4.2) (with $\ell=k, k-1, \ldots)$ to produce a decomposition

$$
\tilde{H}_{k}^{\lambda / \mu}=\bigoplus_{T \in \hat{H}_{k}^{\lambda / \mu}} \tilde{H}_{1}^{T},
$$

where the summands $\tilde{H}_{1}^{T}$ are 1-dimensional vector spaces. This determines a basis (unique up to multiplication of the basis vectors by constants) $\left\{v_{T} \mid\right.$ $\left.T \in \hat{H}_{k}^{\lambda / \mu}\right\}$ of $\tilde{H}_{k}^{\lambda / \mu}$ which respects the decompositions in (4.2) for $1 \leq \ell \leq k$.

Combining (3.1), (3.2) and (3.4) gives that $X^{\varepsilon_{i}}$ acts on the $L(\lambda)$ component of the decomposition (3.10) by the constant

$$
q^{\langle\lambda, \lambda+2 \rho\rangle-\langle\nu, \nu+2 \rho\rangle-\left\langle\varepsilon_{1}, \varepsilon_{1}+2 \rho\right\rangle}=q^{2 c(\lambda / \nu)}
$$

since if $\lambda=\nu+\varepsilon_{j}$, so that $\lambda$ is the same as $\nu$ except with an additional box in row $j$, then $\nu \subseteq \lambda, \lambda / \nu=\square$ and

$$
\begin{aligned}
& \langle\lambda, \lambda+2 \rho\rangle-\langle\nu, \nu+2 \rho\rangle-\left\langle\varepsilon_{1}, \varepsilon_{1}+2 \rho\right\rangle \\
& =\left\langle\nu+\varepsilon_{j}, \nu+\varepsilon_{j}+2 \rho\right\rangle-\langle\nu, \nu+2 \rho\rangle-(1+2(n-1)) \\
& =2 \nu_{j}+\left\langle\varepsilon_{j}, \varepsilon_{j}+2 \rho\right\rangle-2 n+1=2 \nu_{j}+(1+2(n-j))-2 n+1 \\
& =2\left(\nu_{j}+1\right)-2 j=2 c(\lambda / \nu) .
\end{aligned}
$$

Hence,

$$
X^{\varepsilon_{i}} v_{T}=q^{2 c(T(i))} v_{T}, \quad \text { for } 1 \leq i \leq k,
$$

where $T(i)$ is the box containing $i$ in $T$.

The remainder of the proof, including the simplicity of the $\tilde{H}_{k}$-modules $\tilde{H}_{k}^{\lambda / \mu}$, is accomplished as in [R, Thm. 4.1].

(c) The element $X^{\varepsilon_{1}} \cdots X^{\varepsilon_{k}}$ is central in $\tilde{B}_{k}$ (it is a full twist) and hence its image is central in $\tilde{H}_{k}$. The constant describing its action on $\tilde{H}_{k}^{\lambda / \mu}$ follows from the formula $X^{\varepsilon_{i}} v_{T}=q^{2 c(T(i))} v_{T}$.

\subsection{Eigenvalues of the $m_{i}$ in $T_{k}^{a}$}

Let $m_{1}, m_{2}, \ldots, m_{k}$ be the commuting family in the affine TemperleyLieb algebra as defined in (2.15). We will use the results of Theorem 4.1 to determine the eigenvalues of the $m_{i}$ in the (generically) irreducible representations.

THEOREM 4.2. (a) The elements $m_{i}, 1 \leq i \leq k$, mutually commute in $T_{k}^{a}$. 
(b) The eigenvalues of the elements $m_{i}$ are given by the graph $\hat{T}^{/ \mu}$ of (3.17) in the sense that if the set of vertices on level $k$ is

$$
\begin{aligned}
& \hat{T}_{k}^{/ \mu}=\left\{\mu_{1}-\mu_{2}+k, \mu_{1}-\mu_{2}+k-2, \ldots, \mu_{1}-\mu_{2}-k\right\} \cap \mathbb{Z}_{\geq 0} \text {, and } \\
& \hat{T}_{k}^{\lambda / \mu}=\left\{\text { paths } p=\left(\mu=p^{(0)} \rightarrow p^{(1)} \rightarrow \cdots \rightarrow p^{(k)}=\lambda / \mu\right) \text { to } \lambda / \mu \text { in } \hat{T}^{/ \mu}\right\}, \\
& \text { for } \lambda / \mu \in \hat{T}_{k}^{/ \mu} \text { then } \\
& \quad \hat{T}_{k}^{/ \mu} \text { is an index set for the simple } T_{k}^{a} \text { modules } T_{k}^{\lambda / \mu} \\
& \quad \text { appearing in } L(\mu) \otimes V^{\otimes k},
\end{aligned}
$$

and

$$
T_{k}^{\lambda / \mu} \quad \text { has a basis } \quad\left\{v_{p} \mid p \in \hat{T}_{k}^{\lambda / \mu}\right\}
$$

with

$$
m_{i} v_{p}= \begin{cases} \pm\left[p^{(i-1)}+1\right] v_{p}, & \text { if } p^{(i-1)} \pm 1=p^{(i-2)}=p^{(i)} \\ 0, & \text { otherwise }\end{cases}
$$

where $p^{(i)}$ is the partition (a single part in this case) on level $i$ of the path $p$.

(c) $\kappa=m_{k}+[2] m_{k-1}+\cdots+[k] m_{1}$ is a central element of $T_{k}^{a}$ and $\kappa$ acts on $T_{k}^{\lambda / \mu}$ by the constant

$$
\begin{aligned}
{[k] } & \frac{q^{-\left(\mu_{1}+\mu_{2}\right)-\left(\mu_{1}-\mu_{2}\right)+1}}{q-q^{-1}} \\
& +q^{-\left(\mu_{1}+\mu_{2}\right)}\left(\left[\lambda_{1}-\lambda_{2}+2\right]+\left[\lambda_{1}-\lambda_{2}+4\right]+\cdots+\left[\mu_{1}-\mu_{2}+k\right]\right) .
\end{aligned}
$$

Proof. (a) The elements $X^{\varepsilon_{i}}$ commute with one another in the affine Hecke algebra (see (2.4) and the $m_{j}$ are by definition linear combinations of the $X^{\varepsilon_{i}}$ (see 2.15), so they commute.

(b) Let $p$ be a path to $\lambda / \mu$ in $\hat{T}^{\mu}$ and let $T$ be the corresponding standard tableau on 2 rows. If $p^{(i)}=p^{(i-1)}-1=p^{(i-2)}-2$ or If $p^{(i)}=p^{(i-1)}+1=$ $p^{(i-2)}+2$ then $c(T(i-1))=c(T(i))-1$ and, from (2.15) and Theorem $4.1(\mathrm{~b})$,

$$
\begin{aligned}
m_{i} v_{T} & =q^{i-2} \frac{q^{-2 c(T(i))}-q^{-2} q^{-2 c(T(i-1))}}{q-q^{-1}} v_{T} \\
& =q^{i-2} \frac{q^{-2 c(T(i))}-q^{-2} q^{-2 c(T(i))+2}}{q-q^{-1}} v_{T}=0 .
\end{aligned}
$$


If $p^{(i)}=p^{(i-2)}=p^{(i-1)}-1$ with $T^{(i-1)}=(a, b)$ then $c(T(i))=a$ and $c(T(i-1))=b-2$ and

$$
\begin{aligned}
m_{i} v_{T} & =q^{i-2} \frac{q^{-2 a}-q^{-2} q^{-2 b+4}}{q-q^{-1}} v_{T} \\
& =q^{i} \frac{q^{-(a+b+1)}\left(q^{-(a-b+1)}-q^{(a-b+1)}\right)}{q-q^{-1}}=-q^{-m}[a-b+1] v_{T},
\end{aligned}
$$

where $m=|\mu|=a+b-i+1$. If $p^{(i)}=p^{(i-2)}=p^{(i-1)}+1$ with $T^{(i-1)}=(a, b)$ then $c(T(i-1))=a-1$ and $c(T(i))=b-1$ and

$$
\begin{aligned}
m_{i} v_{T} & =q^{i-2} \frac{q^{-2 b+2}-q^{-2} q^{-2 a+2}}{q-q^{-1}} v_{T} \\
& =q^{i} \frac{q^{-(a+b+1)}\left(q^{(a-b+1)}-q^{-(a-b+1)}\right)}{q-q^{-1}}=q^{-m}[a-b+1] v_{T}
\end{aligned}
$$

where $m=|\mu|=a+b-i+1$.

(c) Let $k=|\lambda / \mu|$. The identity

$$
q^{\lambda_{1}+\lambda_{2}-2} \sum_{b \in \lambda / \mu} q^{-2 c(b)}=\left(\sum_{i=\mu_{2}}^{\lambda_{2}-1}\left[\lambda_{1}+\lambda_{2}-2 i\right]\left(q-q^{-1}\right)\right)+[k] q^{\mu_{2}-\mu_{1}+1}
$$

is best visible in an example: With $\lambda=(10,6)$ and $\mu=(4,2)$,

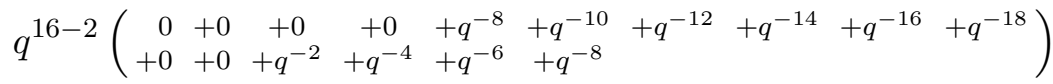

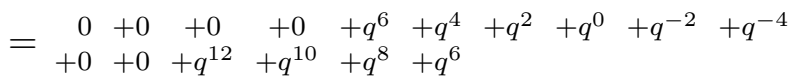

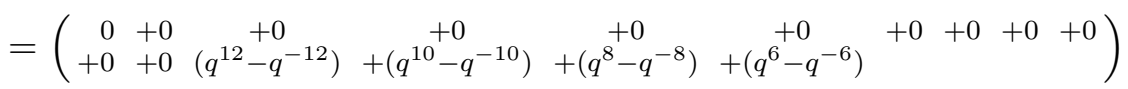

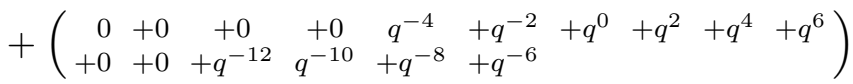

$$
\begin{aligned}
& =\left(\sum_{i=2}^{6-1} q^{16-2 i}-q^{-(16-2 i)}\right)+[10] q^{4-2+1} \text {. }
\end{aligned}
$$

Then Proposition 2.7 says

$$
X^{-\varepsilon_{1}}+\cdots+X^{-\varepsilon_{k}}=q^{-(k-2)}\left(q-q^{-1}\right)\left(m_{k}+[2] m_{k-1}+\cdots+[k] m_{1}\right),
$$


and so $m_{k}+[2] m_{k-1}+\cdots+[k] m_{1}$ acts on $T_{k}^{\lambda / \mu}$ by the constant

$$
\begin{aligned}
& \left(q-q^{-1}\right)^{-1} q^{k-2} \sum_{b \in \lambda / \mu} q^{-2 c(b)}=\left(q-q^{-1}\right)^{-1} q^{-\left(\mu_{1}+\mu_{2}\right)} q^{\lambda_{1}+\lambda_{2}-2} \sum_{b \in \lambda / \mu} q^{-2 c(b)} \\
& =\left(q-q^{-1}\right)^{-1} q^{-\left(\mu_{1}+\mu_{2}\right)}\left([k] q^{\mu_{2}-\mu_{1}+1}+\sum_{i=\mu_{2}}^{\lambda_{2}-1}\left[\lambda_{1}+\lambda_{2}-2 i\right]\left(q-q^{-1}\right)\right) \\
& =[k] \frac{q^{-m-p^{(0)}+1}}{q-q^{-1}}+\sum_{i=\mu_{2}}^{\lambda_{2}-1} q^{-m}[m+k-2 i] \\
& =[k] \frac{q^{-m-p^{(0)}+1}}{q-q^{-1}}+q^{-m}\left(\left[p^{(k)}+2\right]+\left[p^{(k)}+4\right]+\cdots\right. \\
& \left.\cdots+\left[p^{(0)}+k-2\right]+\left[p^{(0)}+k\right]\right)
\end{aligned}
$$

since $\mu_{1}+\mu_{2}=m, \mu_{1}-\mu_{2}=p^{(0)}, \lambda_{1}+\lambda_{2}=m+k$ and $\lambda_{1}-\lambda_{2}=p^{(k)}$.

\section{REFERENCES}

[Ar] S. Ariki, Representations of quantum algebras and combinatorics of Young tableaux, University Lecture Series 26, Amer. Math. Soc., Providence, RI, 2002. ISBN: 0-8218-3232-8, MR1911030 (2004b:17022)

[Ch] I. Cherednik, A new interpretation of Gel'fand-Tzetlin bases, Duke Math. J., 54 (1987), no. 2, 563-577.

[Dr] V. G. Drinfel'd, Almost cocommutative Hopf algebras, Leningrad Math. J., 1 (1990), 321-342.

[Dx] J. Dixmier, Enveloping algebras, Graduate Studies in Mathematics 11, Amer. Math. Soc., Providence, RI, 1996. ISBN: 0-8218-0560-6, MR1393197 (97c:17010)

[FH] W. Fulton and J. Harris, Representation theory. A first course, Graduate Texts in Mathematics 129, Springer-Verlag, New York 1991. ISBN: 0-387-97527-6; 0-38797495-4, MR1153249 (93a:20069)

[GHJ] F. Goodman, P. de la Harpe, and V. Jones, Coxeter graphs and towers of algebras, Mathematical Sciences Research Institute Publications 14, Springer-Verlag, New York, 1989.

[GL1] J. Graham and G. Lehrer, Diagram algebras, Hecke algebras and decomposition numbers at roots of unity, Ann. Scient. Éc. Norm. Sup. $4^{\mathrm{e}}$ série 36 (2003), no. 4, 479-524. MR2013924 (2004k:20007)

[GL2] J. Graham and G. Lehrer, Cellular and diagram algebras in representation theory, Representation theory of algebraic groups and quantum groups, Adv. Stud. Pure Math. 40, Math. Soc. Japan, Tokyo, 2004, pp. 141-173. MR2074593 (2005i:20005)

[Gr] I. Grojnowski, Affine $\mathfrak{s l}_{p}$ controls the representation theory of the symmetric group and related Hecke algebras, arxiv:math.RT/9907129. 
[Ji] M. Jimbo, A q-analogue of $U(\mathfrak{g l}(N+1))$, Hecke algebra, and the Yang-Baxter equation, Lett. Math. Phys., 11 (1986), no. 3, 247-252. MR0841713 (87k:17011)

[K] A. Kleshchev, Linear and projective representations of symmetric groups, Cambridge Tracts in Mathematics 163, Cambridge University Press, Cambridge, 2005. ISBN: 0-521-83703-0, MR2165457 (2007b:20022)

[KL] D. Kazhdan and G. Lusztig, Proof of the Deligne-Langlands conjecture for Hecke algebras, Invent. Math., 87 (1987), no. 1, 153-215. MR0862716 (88d:11121)

[LR] R. Leduc and A. Ram, A ribbon Hopf algebra approach to the irreducible representations of centralizer algebras: the Brauer, Birman-Wenzl, and type A IwahoriHecke algebras, Adv. Math., 125 (1997), 1-94. MR1427801 (98c:20015)

[Mac] I. G. Macdonald, Symmetric functions and Hall polynomials, Second Edition, Oxford University Press, 1995.

[OR] R. Orellana and A. Ram, Affine braids, Markov traces and the category O, Proceedings of the International Colloquium on Algebraic Groups and Homogeneous Spaces, Mumbai 2004, Tata Institute of Fundamental Research (TIFR), Mumbai, Narosa Publishing House, 2007, pp. 423-473.

[OV] A. Okounkov and A. Vershik, A new approach to representation theory of symmetric groups, Selecta Math. (N.S.), 2 (1996), no. 4, 581-605. MR1443185 (99g:20024)

[R] A. Ram, Skew shape representations are irreducible, Combinatorial and Geometric representation theory (S.-J. Kang and K.-H. Lee, eds.), Contemp. Math. 325, Amer. Math. Soc., 2003, pp. 161-189. MR1988991 (2004f:20014)

[Re] N. Yu. Reshetikhin, Quantized universal enveloping algebras, the Yang-Baxter equation, and invariants of links I, LOMI Preprint E-4-87 (1987).

Tom Halverson

Department of Mathematics

Macalester College

Saint Paul, MN 55105

U.S.A.

halverson@macalester.edu

Manuela Mazzocco

Department of Mathematics

University of Wisconsin

Madison, WI 53706

U.S.A. 
Arun Ram

Department of Mathematics and Statistics

University of Melbourne

Parkville VIC 3010

Australia

aram@unimelb.edu. au

and

Department of Mathematics

University of Wisconsin

Madison, WI 53706

U.S.A. 